\title{
EL PIEDEMONTE PLIOCUATERNARIO EN EL SECTOR CENTRAL PIRENAICO (HUESCA Y LERIDA)
}

\author{
$\mathbf{P} \quad \mathbf{O} \mathbf{R}$ \\ Alderto, F.1 ; Gu'térrez, M. ${ }^{2}$; Ibáñ̄ez, M.-J.3; Machin, J. ${ }^{1}$; \\ Mel.éndez, A. ${ }^{4}$; Peña, J. L. ${ }^{5}$; Pocovi, A. ${ }^{6}$; Rodríguez, J. ${ }^{6}$
}

\section{R ES U M E N}

Se estudian los materiales pliocuaternarios del sector Central Pirenaico, realizando un análisis de los mismos en los interfluvios Segre-Cinea y Cinca-Gállego. Se trata de tres niveles de acumulación detritica, de desigual desarrollo, que presentan caracteristicas de depósitos de abanicos aluviales, con modelado en glacis, previo a la implantación de la red fluvial cuaternaria. Se realiza igualmente la descripción y características de los suelos y niveles de costras desarrollados sobre las acumulaciones aluviales.

\section{S U M M A R Y}

The paper deals on the study of plioquaternary materials from Central pyrenaic area in base of their analysis in the interareas of rivers Segre-Cinca and Cinca-Gallego. Three inequally developped levels of detritic aeumulation, display, in typical features of alluvial fan deposits with glacis landform prior to the setting of the fluvial net, have been recognised. Soils and crust levels developped on aluvial accumulations are also described and cha. racterised.

1 Estación Experimental Aula Dei. C. S. I. C. Zaragoza.

2 Cátedra de Geología. Facultad de Ciencias. Zaragoza.

3 Departamento de Geografía. Facultad de Filosofía y Letras. Zaragoza.

4 Departamento de Estratigrafía y Geología Histórica. Facultad de Ciencias. Zaragoza.

5 Departamento de Geografía. Colegio Universitario. Teruel.

6 Departamento de Geomorfologia y Geotectónica. Facultad de Ciencias. Zaragoza. 


\section{INTRODUCCION}

Los materiales detríticos pliocuaternarios del piedemonte pirenaico aparecen modelados en glacis en áreas interfluviales; están desconectados de la red hidrográfica actual y topográficamente se sitúan por encima de las terrazas fluviales cuaternarias. Por su morfología superficial y estructuras internas se identifican con depósitos de abanicos aluviales, encostrados en superficie, lo que ha favorecido su conservacón en el relieve actual.

Su altitud relativa sobre la red fluvial actual, morfología espacial, grado de encostramiento y semejanza de facies con otras formaciones peninsulares se puede asignar una edad pliocuaternaria para éstas acumulaciones detríticas.

Los depósitos estudiados se ubican en el somontano oscense y parte del sector leridano, estando por lo tanto situados en la margen septentrional de la Depresión del Ebro, entre los relieves calcáreos de las sierras exteriores pirenaicas, al norte, y las plataformas calizas de las muelas del centro de la Depresión (Almudévar, Alcubierre, Sigena, etc...).

El estudio se ha limitado a las áreas interfluviales comprendidas entre el Gállego y Segre, como zona más representativa de los depósitos pliocuaternarios, aunque también existen restos de estos materiales al Oeste del Gállego (Monlora) y Este del Segre (Llanos de Urgell y La Segarra) y también en el área de Candasnos, al pie de la Sierra de Ontiñena.

El Pliocuaternario se asienta sobre los materiales del Terciario continental de la Depresión del Ebro, constituida sobre los yesos del Anticlinal de Barbastro-Balaguer, areniscas y limolitas rojas de la Formación Peraltilla y areniscas, margas y conglomerados ocres de la Formación Sariñena (Quirantes, J., 1978). Exceptuando el núcleo del Anticlinal de Barbastro-Balaguer, la deformación es escasa, presentando una estructura suavemente ondulada.

Por otra parte, los materiales sobre los que se asientan los depósitos plio. cuaternarios son muy poco resistentes a la erosión, en contraste con los niveles calcáreos de las muelas y las calizas y conglomerados de las sierras exteriores, limites meridional y septentrional respectivamente, lo cual fue un condicionante para las génesis de un área deprimida.

Ya una serie de autores se han preocupado del estudio de estos depósitos Panzer, W. (1926) señala que las terrazas más altas $(100-115 \mathrm{~m}$.) pertenecen al Plioceno más joven o al diluvial antiguo. Solé Sabarís, L. y Font Tullot, J. M. (1929) ponen de manifiesto terrazas aluviales anteriores al Cuaternario entre 120 y $250 \mathrm{~m}$. de altura sobre el Segre. En el piedemonte oscense BarreRE, P. (1951) define los niveles "corona" como precuaternarios. La única datación existente en la Depresión del Ebro para el Pliocuaternario es la efectuada por Crusafont, M., Villalta, J. M. y Riba, O. (1957) en Villarroya (Logroño) mediante una fauna de mamíferos que proporciona una edad Villafranquiense, a la cual hace referencia BoMER, B. (1957 y 1979) para considerar como Villafranquiense los niveles aluviales más altos de la Depresión del Ebro. Mensua, S. e IbáÑez, M. J. (1977) efectúan una cartografía de los conos y abanicos pliocuaternarios del centro de la Depresión del Ebro. En el 
piedemonte leridano Peña Monne, J. L. (1980) señala glacis pliocuaternarios y en el somontano oscense RoDríguez VIDAL, J. (1981) hace referencia a una red de abanicos aluviales húmedos de edad pliocuaternaria.

\section{CARACTERISTICAS DEL PLIOCUATERNARIO}

Los depósitos pliocuaternarios se encuentran distribuidos en retazos de extensión variable, a causa de los procesos de incisión que han tenido lugar fundamentalmente en el transcurso del Cuaternario. Sin duda, el área en los que aparecen más claramente representados, tanto por su amplitud como por las relaciones que se pueden establecer entre ellos, está situada en la margen derecha del Noguera Ribàgorzaná. Por el contrario, el resto de la zona está caracterizada por vestigios de estos materiales relegados, en muchos casos, a cerros testigos, apoyados generalmente en un substrato areniscoso, en los que resutla difícil establecer correlaciones entre los distintos afloramientos, hasta llegar prácticamente a desaparecer en la margen derecha del Gállego.

Las distintas observaciones efectuadas han puesto de manifiesto la existencia de tres niveles de acumulación con características de abanicos aluviales modelados en glacis, situados por encima de! nivel más alto de las terrazas encajadas en los ríos principales, lo que nos lleva a considerar que se trata de niveles pliocuaternarios emplazados antes de que haya tenido lugar la jerarquización de la red fluvial cuaternaria.

La cartografía de estos materiales y la pendiente de sus superficies indica, lógicamente, una procedencia de las sierras pirenaicas. Por otra parte, es de destacar el enlace existente, en algunos casos, entre los depósitos pliocuaternarios y niveles de arrasamiento presentes en el anticlinal de Barbastro-Balaguer y que, en ocasiones (área de Alfarrás y Abiego), conservan restos de acumulaciones de esta edad.

Para efectuar un análisis de los niveles pliocuaternarios hemos estimado conveniente considerar por separado los interfluvios Segre-Cinca y Cinca-Gállego. La razón de esta diferenciación es la de que en este último no se observan en el depósito cantos de procedencia del Pirineo axial.

\section{II.1. Fl interfluvio Segre-Cinca}

Ya hemos indicado anteriormente que es en esta zona donde los materiales pliocuaternarios conservan su máxima extensión, quedando enmarcados entre el Noguera Ribagorzana y el Arroyo de la Clamor. La cartografía permite diferenciar tres niveles, encajados entre sí. El nivel más antíguó se extiende desde Alguaire hasta el cerro de Valseca, existiendo otros retazos al Oeste de este afloramiento. El nivel medio queda fundamentalmente situado al Oeste del nivel superior, y es el más bajo el que alcanza una mayor superficie, alargándose a una franja continua desde Alfarrás hasta el Este do Fraga, a lo largo de $36 \mathrm{Kms}$. En Plá de Valmanya (NE de la Hoja 387) èl 
nivel inferior está $38 \mathrm{~m}$. por debajo del nivel medio y éste, a su vez, $16 \mathrm{~m}$. bajo el medio y este último $10 \mathrm{~m}$. en el superior.

La diferenciación de los niveles pliocuaternarios con respecto a las terrazas la efectuamos por criterios morfológicos de pendiente del depósito, distribución espacial $\mathrm{y}$, en la mayor parte de los casos distinta dirección de este modelado con respecto a las terrazas.

La pendiente de la superficie es bastante uniforme para todos los niveles y fluctúa entre el 5 y 6 por mil. El sentido de los mismos es hacia el SSW para los depósitos más continuos, que arrancan del área de Alfarrás y hacia el S y SSE para los restantes. Estas observaciones parecen indicar que existieron, para estas épocas, dos abanicos aluviales, cuyo ápice debía estar situado en la zona de tránsito de las Sierras Exteriores y la Depresión del Ebro, ubicándose en la proximidad de los actuales rios Noguera Ribagorzana y Cinca.

El espectro litológico de los materiales constituyentes de estos tres niveles es muy similar. Se han reconocido cantos de caliza, arenisca, cuarcita, esquisto, conglomerado triásico, roca volcánica, granito, etc. El tamaño máximo de los cantos es de $50 \mathrm{~cm}$. Los cantos de granito presentan, en muchos casos, una intensa alteración que los hace disgregables. Los de caliza, sobre todo los situados a techo del depósito, manifiestan una profuda disolución que los convierte en formas lenticulares.

La potencia de los depósitos es variable, dependiendo de su ubicación dentro del abanico de Alfarrás, la potencia del nivel superior alcanza los $20 \mathrm{~m}$. mientras que en la Dehesa de la Sardera $(2 \mathrm{kms}$. al oeste de Villanueva de Alpicat) el espesor se reduce a unos $2 \mathrm{~m}$. La elevada potencia existente en Alfarrás corresponde, sin duda, a la zona apical del abanico. Los espesores de los niveles inferiores han sido medidos en áreas distales de los abanicos. El nivel medio, en la Sierra de Coscollar (carretera de Valmaña-Almacellas), tiene una potencia superior a los $3,5 \mathrm{~m}$. Finalmente, el nivel más bajo en la Sierra Pedregosa (Este de Fraga) posee un espesor variable comprendido entre 2 y $6 \mathrm{~m}$.; en Almacellas es de $3,5 \mathrm{~m}$.

La relación de los depósitos con el sustrato es claramente erosiva y, a veces, canalizada. Estas circunstancias aparecen muy manifiestas en Sierra Pedregosa y son la causa de la variación de potencia existente. Los niveles plio. cuaternarios presentan características sedimentológicas similares, entre las que destacan cuerpos canaliformes con relleno de gravas que presentan imbricación de cantos y granoselección positiva, y cuñas arenosas con estratificación cruzada planar y superficies de reactivación. Son frecuentes los cambios texturales. Esta disposición corresponde a una asociación de canales y barras en un contexto tipo braided (fig. 1).

Estos depósitos presentan a techo un fuerte encostramiento y están coronados por costras calizas acintadas de potencia variable. Estas costras se describirán más detalladamente en un apartado posterior.

Los bordes de los afloramientos de estos niveles presentan frecuentemente pequeñas cornisas resultantes de la resistencia a la erosión de la costra caliza somital y las vertientes a su pie están, casi en su totalidad, recubiertas por 


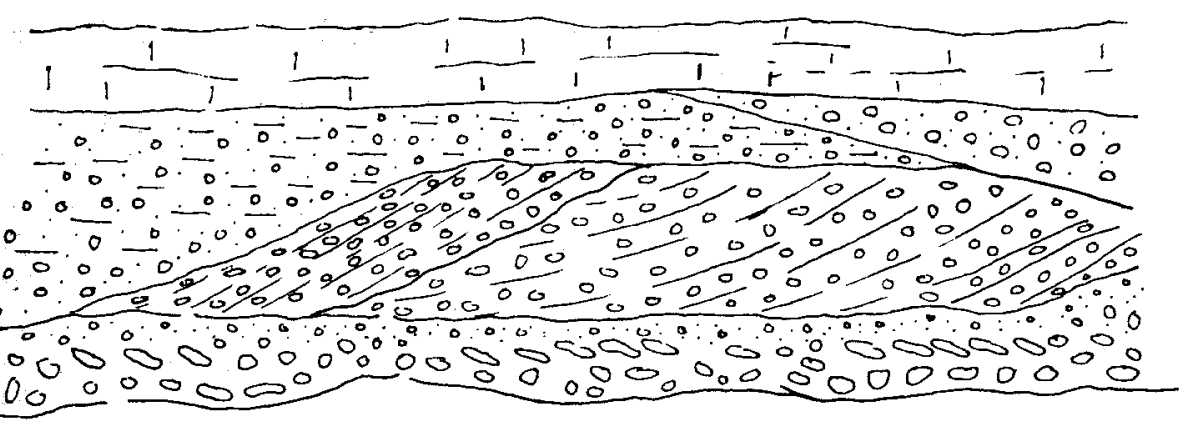

Desarrollo de barra longitudinal. Sierra Pedregoso.

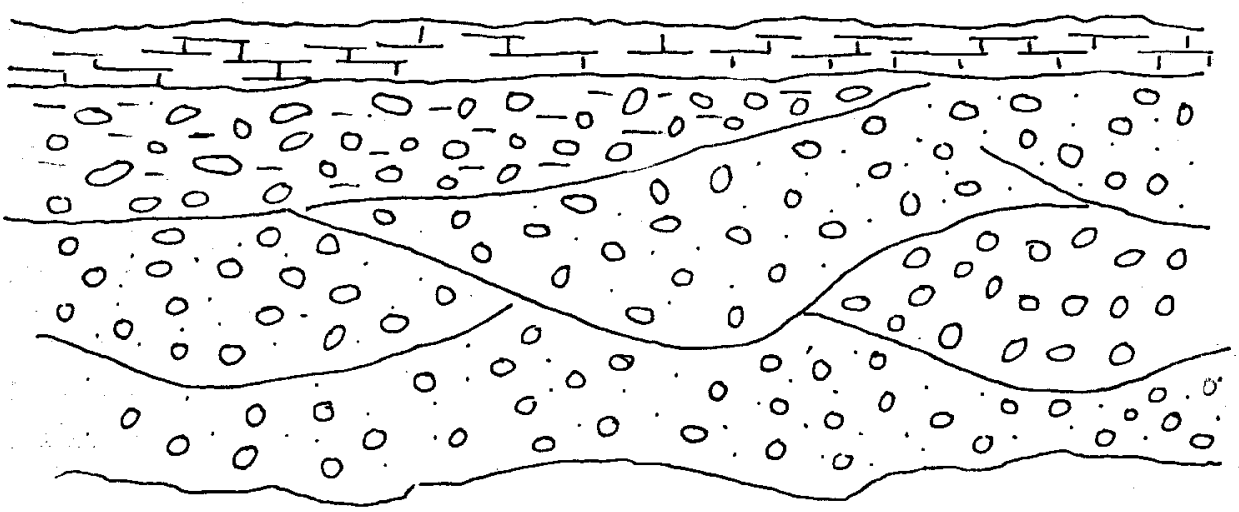

Cuerpos canaliformes con relleno masivo de gravas. Canales entrelazados. Sierra Pedregoso.

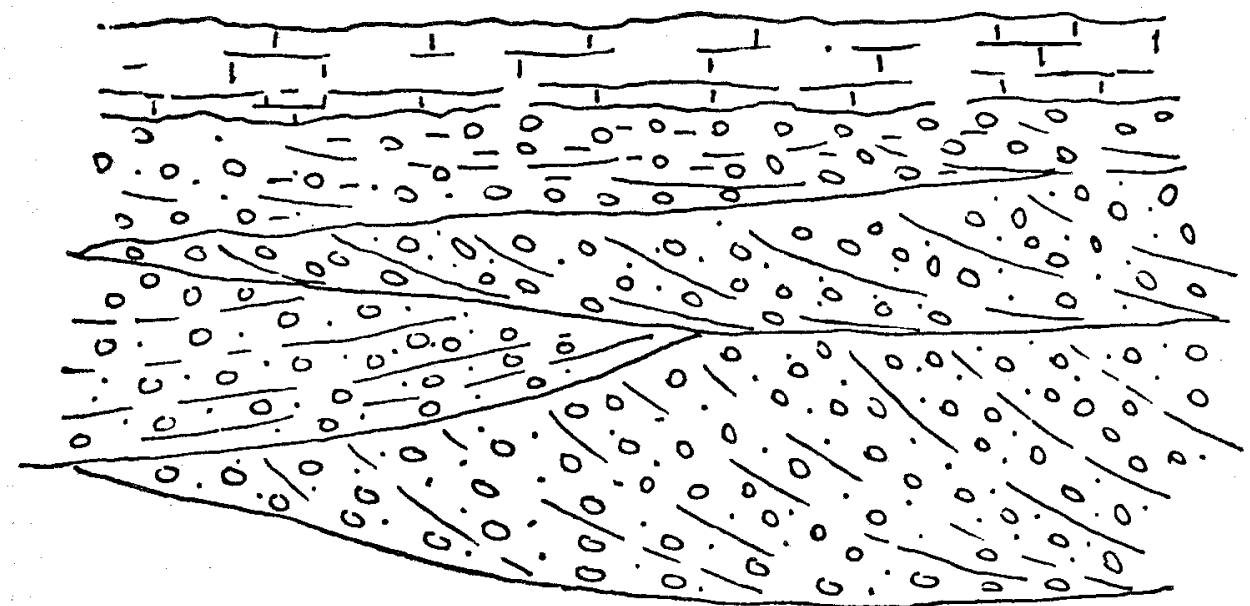

Gravas con estratificación cruzada en surco. Fontanilla.

Fig. 1.

Depósitos Braided de gravas, con desarrollo de costras en la parte superior. 
detritos que la regularizan. La superficie de los niveles está surcada por una red tenue de valles de fondo plano.

Los relieves estructurales del anticlinal Barbastro-Balaguer presentan un arrasamiento generalizado, acompañado en numerosos casos por materiales detríticos. Tanto su arrasamiento como su cobertera empalman claramente con el nivel superior pliocuaternario. Por otra parte, estos piedemontes, que se desarrollan en la región La Litera-Alfarrás, presentan débiles ondulacionés locales debidas a la tectónica de los yesos infrayacentes (PEÑ̈̃. J. L., 1980), pero no se han reconocido importantes levantamientos similares a los descritos más al Este, en las inmediaciones del Balaguer (Solé SABARís, 1953).

Uno de los problemas que se plantean en la diferenciación de los niveles inferiores pliocuaternarios, es su relación con los niveles superiores de las terrazas de las arterias principales. En nuestro caso, esta disquisición se plantea al Este de Albalate de Cinca (fig. 2).

Aquí se pueden reconocer diez niveles aterrazados, a las siguientes alturas relativas aproximadas sobre el río Cinca: $2 \mathrm{~m}$., $10 \mathrm{~m} ., 20 \mathrm{~m} ., 40 \mathrm{~m}$., $60 \mathrm{~m}$., $90 \mathrm{~m} ., 120$ m., 150 m., 200 m. y 220 m. Mientras los dos niveles más elevados no resultan problemáticos en su adscripción al Pliocuaternario, pese a su posición geomorfológica aislada, sin embargo, el nivel de $150 \mathrm{~m}$. se presenta adosado a las terrazas cuaternarias en posición más incierta. No obstante, más al Sur se pone de manifiesto una relación topográfica con el nivel Pliocuaternario inferior del Noguera Ribagorzana, al N de Fraga, lugar en el cual empalmarían ambas corrientes fluviales en tales momentos. Con posterioridad, el Noguera Ribagorzana abandonó definitivamente esta zona para dirigirse hacia el Segre, ya durante todo el Cuaternario.

La separación entre niveles pliocuaternarios y terrazas cuaternarias presenta un cierto confusionismo, dado que existen además acumulaciones de glacis y vertientes de empalme, actualmente desprovistas de conexion con el área madre donde estarían enraizadas. Tales glacis deben considerarse como cuaternarios, pues se apoyan sobre la terraza superior $(120 \mathrm{~m})$ del rió Cinca o convergen en Peñarroya, al E. de Sant Salvador, con otros glacis de procedencia más oriental formando un amplio valle en cuna, cuyo fondo se sitúa a alturas similares a las de la terraza superior.

\section{II.2. El INTERfluviọ Cinca-Gállego}

En general, los afloramientos de los niveles pliocuaternarios en este área se encuentran más disectados por la red fluvial y como consecuencia aparecen más diseminados, como por ejemplo, las elevaciones interfluviales de la Hoya de Huesca. Al Oeste del Gállego, el desarrollo del Pliocuaternario es muy reducido y únicamente hemos reconocidó el afloramiento del cerro testigo de Monlora, coronado por una potente costra caliza.

Al igual que en el interfluvio Segre-Cinca, se reconocen en esta zona tres niveles pliocuaternarios encajados. Por lo general, no suelen aparecer localmente continuos los tres niveles, excepto en el área Antillón-Novales. Normal- 


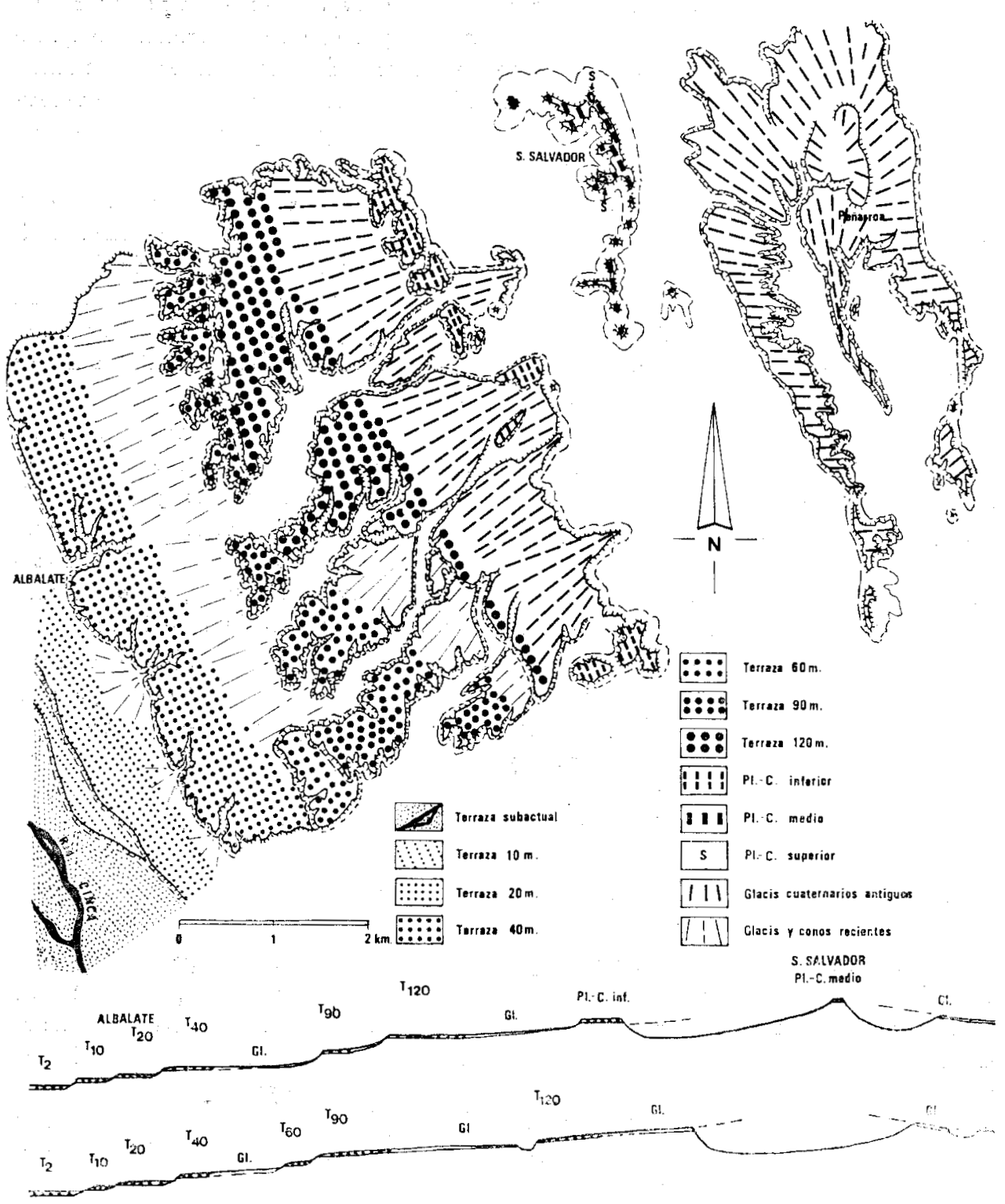

Fig. 2. - Terrazas Cuaternarias y niveles Plio-Cuaternarios del valle del Cinca en Albalate (Huesca). 
mente sólo suelen presentarse dos. Ya hemos señalado que no existe una gran continuidad en afloramiento de los mismos, salvo el nivel superior en Berbegal que alcanza una dimensión máxima de $20 \mathrm{kms}$.

En el área de Berbegal el nivel medio está encajado en el superior $35 \mathrm{~m}$. y el bajo sobre el medio de 7 a $10 \mathrm{~m}$. Por otra parte, en el Estrecho de Quinto (al Este de Huesca) el nivel más alto de terrazas del río Flumen se encuentra situado $35 \mathrm{~m}$. por debajo del nivel inferior pliocuaternario. Las pendientes medidas en numerosos puntos fluctúan entre 8 y 10 por mil. El sentido de la misma varía entre NW-SE y NE-SW.

El estudio de los diferentes afloramientos permite reconocer para este interfluvio un sistema de cinco importantes abanicos aluviales. El área apical de cuatro de ellos se encuentra situada en las zonas de salida de los ríos Riel, Isuela, Calcón y Alcanadre-Balcés. El otro está desconectado de la red fluvial actual, como apunta BoMER, B. (1979), y perteneció a la salida del antiguo río Vero al pie del Anticlinal de Barbastro-Balaguer, tal como se deduce del conjunto de terrazas de este río.

La litología de los cantos constituyentes de estos niveles es totalmente de procedencia prepirenaica con dominio de calizas eocenas. Puntualmente hemos reconocido muy escasos cantos de granito muy meteorizado y de cuarcita, que interpretamos como que proceden de los conglomerados del Eoceno superior de la Formación Campodarbe de Puigdefábregas, C. (1975). También señalan la presencia de cantos del Pirineo axial Mensua, S. e Ibáñez, M. J. (1977) y BoMER, B. (1979) para el pliocuaternario de este interfluvio.

La potencia del nivel alto en Berbegal es de $3 \mathrm{~m}$. y más al Sur alcanza $6 \mathrm{~m}$. en la Estación del Tormillo (Fontanetas). El nivel medio tiene algo más de $1 \mathrm{~m}$. en Peralta de Alcofea, mientras que en áreas proximales pueden medirse $18 \mathrm{~m}$. en la Corona de Arascués y $12 \mathrm{~m}$. en la presa de Guara (Barranco Calcón). Finalmente, el nivel bajo alcanza una potencia de $13,5 \mathrm{~m}$. en la Sarda de Bandaliés y de 2,5 m. en el Pico Torraza (carretera Huesca-Ayerbe).

Las características sedimentológicas de estos materiales, las costras acompañantes, así como los rasgos geomorfológicos, son semejantes a las del interfluvio Segre-Cinca descritos en el apartado anterior.

El anticlinal Barbastro-Balaguer presenta un arrasamiento general y esta superficie empalma con el nivel pliocuaternario inferior (Abiego-Peraltilla), a diferencia del interfluvio Segre-Cinca donde enlaza con el nivel superior. Además, se observan aplanamientos locales en las calizas de las Sierras Exteriores (entre San Julián de Banzo y Santa Eulalia la Mayor) que buzan hacia la Depresión y empalman con el nivel inferior pliocuaternario. Estos últimos arrasamientos no poseen material detrítico y se presentan karstificados con terras rossas acompañantes.

Los niveles medio y superior presentan una desconexión con la red fluvial actual y una disposición que permite reconstruir, ${ }^{,}$aunque en algunós casos cón dificultad, una antigua morfología de abanicos. El nivel inferior posee esta morfología en las áreas apicales, mientras que aguas abajo los testimonios se 
distribuyen en bandas más o menos paralelas a los cursos actuales, lo que indica un comienzo de jerarquización de la red.

\section{II.3. LAS costras}

\section{Sector al Este del Cinca}

En esta área los encostramientos tienen un desarrollo comprendido entre 0,5 y $1,5 \mathrm{~m}$., y se sitúan sobre niveles de gravas. Se pueden diferenciar tres niveles: Nivel inferior de costra pulverulenta no compacta. Nivel medio formado por costra de aspecto noduloso, con abundantes fragmentos, agregados y con frecuencia recrecimientos en la base de los clastos. Nivel superior de costras laminares con presencia de fragmentos, agregados y laminaciones continuas e irregulares (fig. 3. Sierra Pedregosa, Almacellas).

Las muestras de niveles superiores, muestras 1,2 y 3, tanto de costras laminares como nodulosas se caracterizan por un bajo contenido en residuo insoluble $(4.8 \%, \mathrm{y}$ simultáneamente una alta relación $\mathrm{Ca} / \mathrm{Mg}$, superior a 40/1. (Cuadro I). En estas muestras se han observado los siguientes elementos constituyentes y estructuras de orden interno: fragmentos y agregados calcáreos, ooides, concrecciones en la base de los clastos y agregados-laminaciones micríticas fragmentadas, microcavidades, laminación micrítica irregular y continua. Estos elementos se encuentran ordenados dentro del nivel superior de manera que en la base encontramos fragmentos y agregados de aglutinación, ooides y generalmente un recubrimiento de laminación micrítica frecuentemente fracturada. Por encima se encuentran fragmentos y agregados con recrecimientos basales, asociados a laminaciones micríticas irregulares y discontinuas, observándose algunas superficies irregulares con microcavidades. A techo se desarrolla generalmente una costra laminada que homogeiniza la superficie, con una disposición abultada irregularmente.

\section{Sector al Oeste del Cinca}

Los encostramientos observados en este área son diferentes en cuanto a su disposición. No se identifican los tres niveles como en el caso anterior, sino que se trata de una sucesión de potencia similar y más o menos compleja de niveles de costras laminadas y costras nodulosas a veces con aspecto brechoide, encontrándose cantos cuarcíticos (fig. 3. Berbegal).

Las muestras estudiadas 5,6 y. 8 y sobre todo las correspondientes a costras laminadas presentan un residuo insoluble superior a las anteriores $(17-22 \%)$ y como en el caso anterior una alta relación $\mathrm{Ca} / \mathrm{Mg}$. Con porcentajes de Ca entre $20.30 \%$ de $\mathrm{Mg}$ siempre inferiores al $1 \%$ (cuadro I). En estas muestras se han observado agregados con abundantes concrecciones de recrecimiento, estructuras alveolares, nódulos micritizados, superficies con microcavidades, laminaciones micríticas, desordenadas e irregulares y presencia de clastos cuarcíticos.

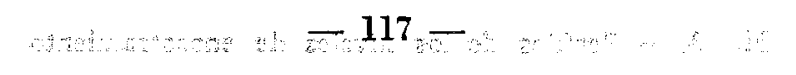




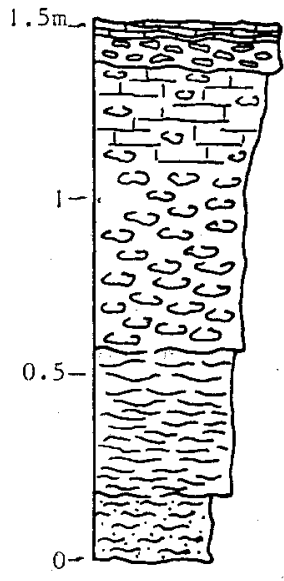

Nivel de costra laminada con intraclastos

Costra nodulosa compactada

Nivel noduloso debilmente compactado

Nivel bandeado con laminaciôn nodulosa

Nivel pulverulento debilmente compactado

\section{ALMACELLAS}

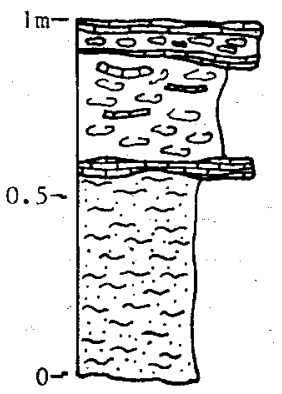

Nivel de costra laminada

Costra nodulosa con intraclastos

Nivel de costra laminada

Nivel de costra pulverulenta

BERBEGAL

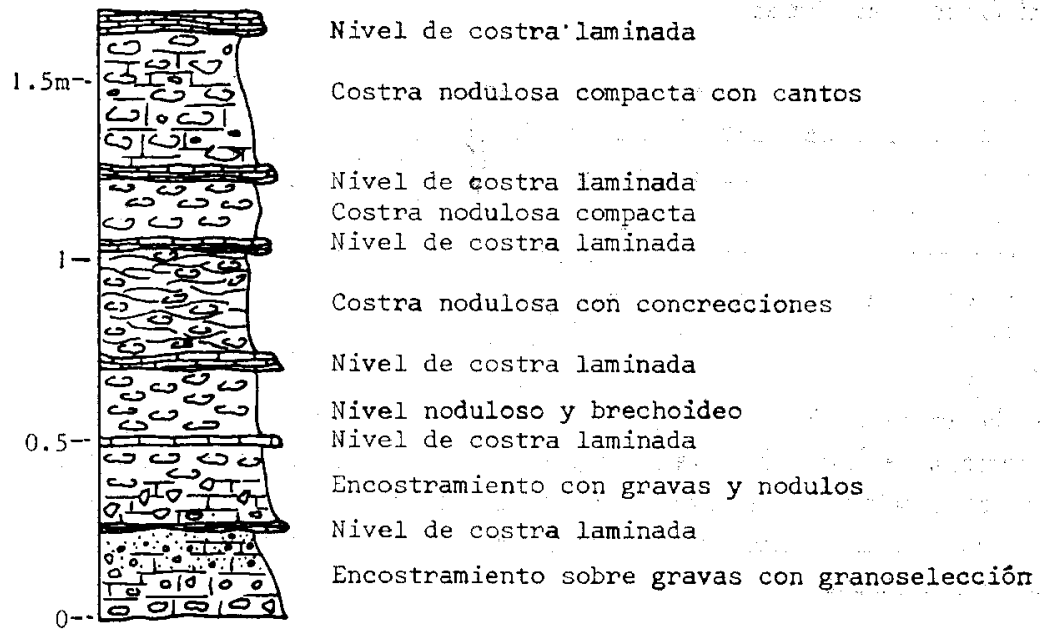

Fig. 3. - Perfiles de los niveles de encostramiento. 


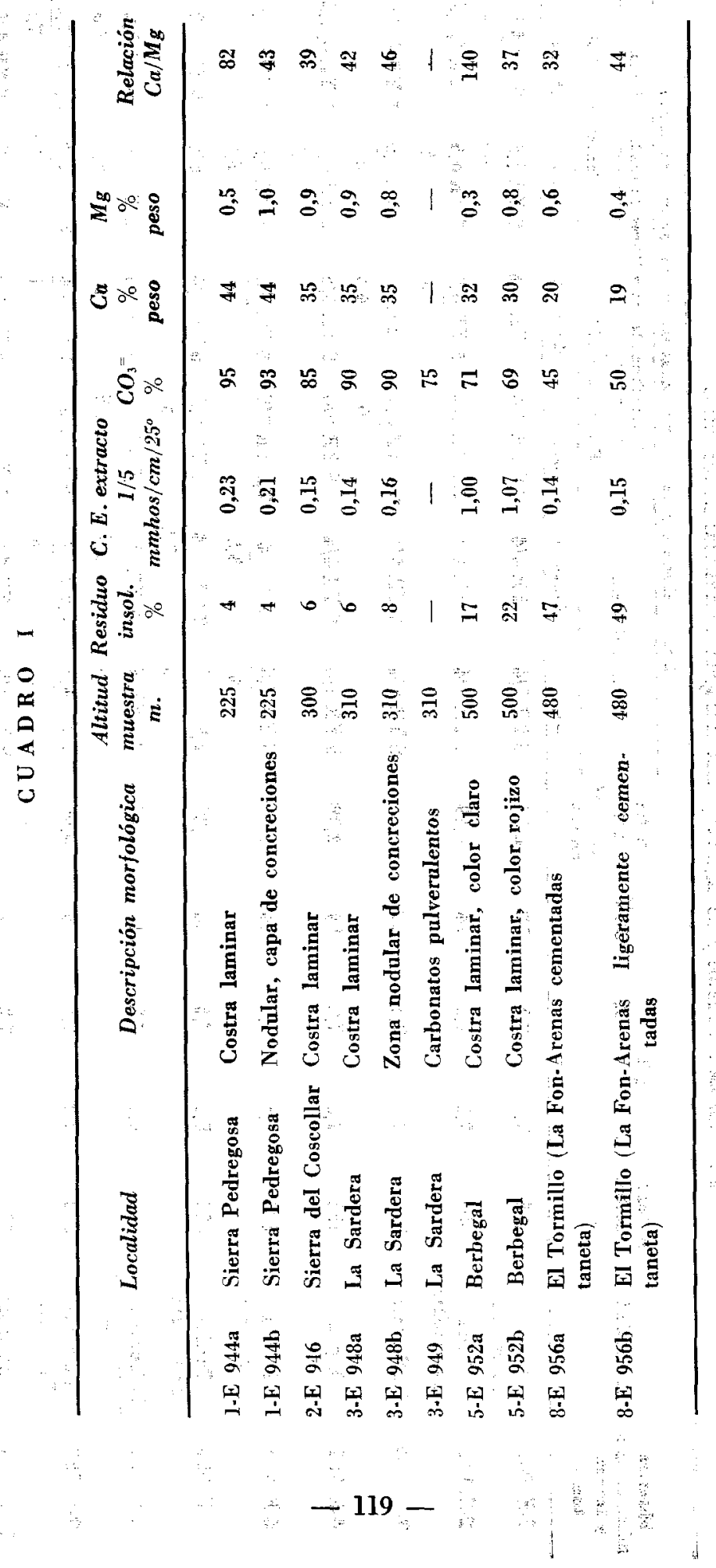




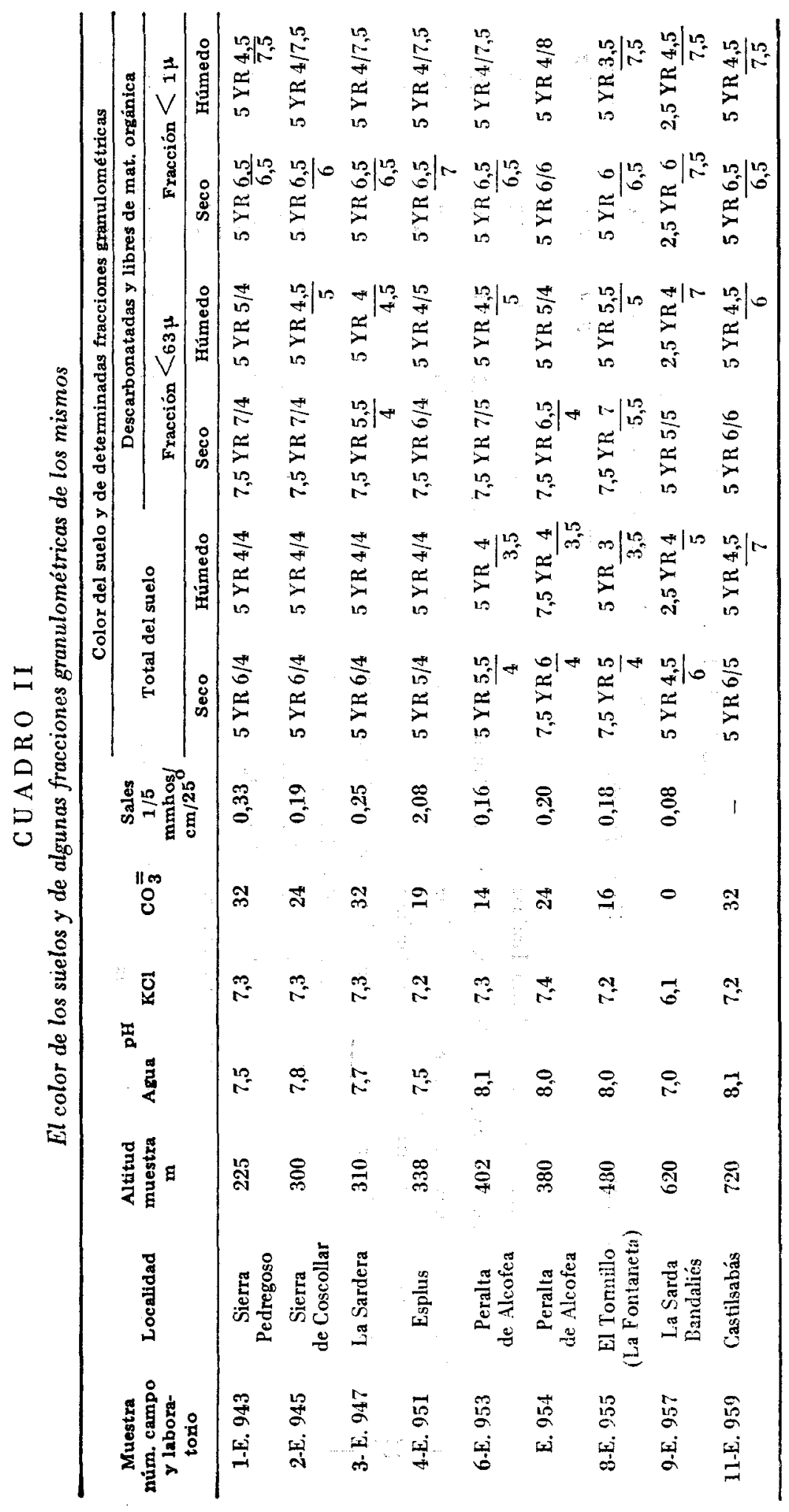



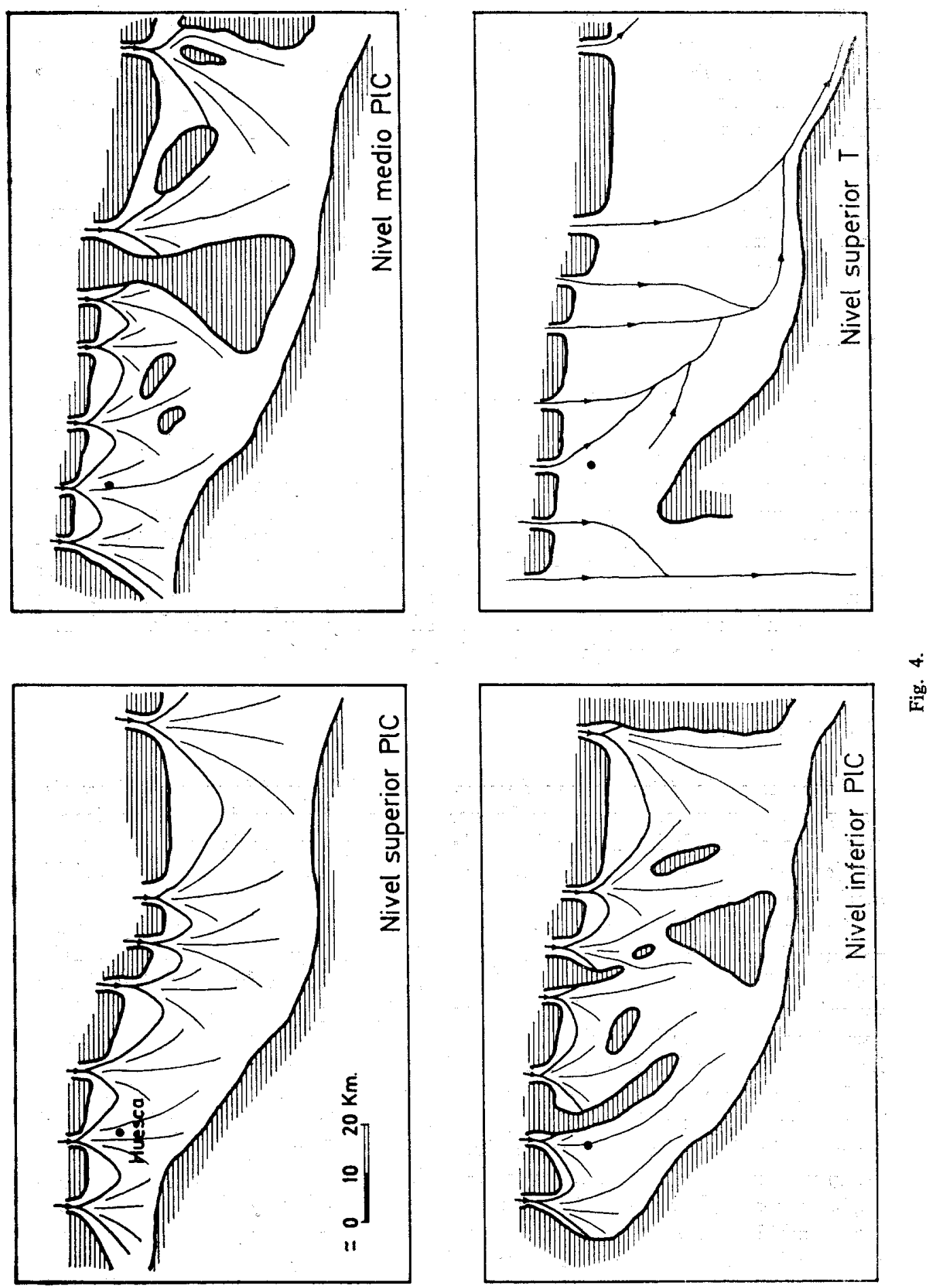
Estos elementos están mal ordenados en una sucesión compleja, en la que en la base y dentro de una matriz micrítica porosa se incluyen litoclastos y agregados con abundantes recrecimientos, nódulos formados a partir de la matriz con estructura alveolar. Sobre éstas se desarrolla un tapizado con laminación micrítica y sobre él se generan superficies irregulares con microcavidades.

La parte superior presenta agregados y fragmentos con algunos clastos cuarcíticos con laminación micrítica desordenada, por encima se sitúa una laminación micrítica irregular, dentro de la cual existen superficies irregulares que dividen al conjunto en distintos niveles diferenciados.

\section{Procesos}

Los procesos individuales que podrían haber dado lugar a los distintos elementos y estructuras observadas deben entenderse en un contexto climático determinado : condiciones de aridez y sus lógicas variaciones. Podrían corresponder a procesos de aglutinación, estabilización de granos, disolución, desesación, acumulación de partículas y precipitación por evaporación.

Parece importante separar los procesos de precipitación inicial de los carbonatos, de su posterior diagénesis, en particular los referentes a las costras laminadas, cuya formación podría actuar de techo impermeable, fosilizando la parte inferior aislándola de procesos edáficos y favoreciendo la implantación de una red de drenaje superficial que erosionaría el suelo.

\section{II.4. Los suelos}

Sobre estos depósitos se han desarrollado suelos, genéticamente influenciados por los mismos, fundamentalmente a través de tres factores de forma. ción, la geología de los materiales de la parte superior de la unidad, el tiempo (evidentemente el suelo ha de ser más joven que la unidad geomorfológica) y la topografía.

La topografía de la unidad y la presencia de costra caliza en la parte superior del paquete sedimentario ha condicionado, seguramente con la complicidad de fluctuaciones climáticas de poca amplitud, la conservación de las superficies originales y simultáneamente de alguna porción de los suelos. Otra parte importante de los suelos ha sido arrastrada por la red de drenaje super. ficial exigida por la impermeabilidad del nivel de costra.

Los materiales de los suelos presentan respecto a los desarrollados sobre las terrazas altas unos hues más rojos predominio de hues 5 YR que sólo en el suelo de Bandaliés pasa a alcanzar hues de 2,5 YR en alguna de las fracciones granulométricas. La destrucción de carbonatos con $\mathrm{HCl} 0.1 \mathrm{~N}$ y de materia orgánica con $\mathrm{H}_{2} \mathrm{O}_{2}$ hace derivar los colores hacia values y chromas más altos pero también hacia una mejor percepción del hue correspondiente (eyidenciado en la constancia de hues entre colores en seco y húmedo).

$$
=122=
$$


Los materiales groseros sedimentarios y la misma costra caliza han actuado como roca madre de los suelos lo que se pone de manifiesto en los fenómenos de disolución de los cantos de caliza (disueltos hasta aparecer con sección planoconvexa, con la convexidad en la parte inferior del canto) y en los procesos de disolución y reprecipitación de los carbonatos de la costra desarrollando estructuras laminares. A nivel morfológico, este proceso en que los cantos actúan como roca madre de los suelos comienza a ser patente a partir del nivel de la terraza III. En los sedimentos pliocuaternarios resulta muy aparente especialmente en Bandaliés en donde el color rojo del suelo puede proceder de las arcillas heredadas y en el que la intensidad del proceso es congruente con la descarbonatación que presenta la fracción fina del suelo.

En las otras superficies los suelos contienen carbonato cálcico en su matriz, no diferenciable en primario o secundario. Estos extremos deberían estudiarse más minuciosamente.

De estudios realizados en los materiales aluviales del Gállego y del Cinca la altitud a que se encuentra la línea de descarbonatación de los primeros $25 \mathrm{~cm}$. de los suelos se sitúa entre los $850-900 \mathrm{~m}$., para suelos cultivados.

Este límite altitudinal indudablemente se rebajará para suelos no utilizados agronómicamente. Por la estrecha relación existente al N. del Ebro entre altitud y condiciones climáticas (temperatura y pluviometría) parece evidente que los suelos de Castilsabás tienen que haber estado descalcificados alguna vez y habrá relictos en bolsones, testigos de esta descalcificación. Los actuales valores de carbonatos pueden corresponder a una calcificación secundaria procedente de la costra.

La localización de áreas de coincidencia de costra caliza con suelos descarhonatados puede ser interesante para comprender la evolución climática durante el Cuaternario en la Depresión del Ebro.

\section{EVOLUCION}

En las Sierras Exteriores oscenses Barrere, P. (1951), reconoce dos superperficies de erosión, la denominada superficie superior de Sierra de Guara y otra, más baja, que corresponde a la superficie senil general de las Sierras, con pendientes al Sur y al Oeste, respectivamente. Posteriormente, BarreRE, P. (1962) en el estudio del Pirineo navarro oriental, señala una fase principal de denundación que genera una superficie que la considera como "finimiocena, sin duda pontiense". PẼ̃A, J. L. (1978, 1979 y 1980) en sus trabajos sobre el Pirineo leridano diferencia una superficie principal en las Sierras Interiores y Exteriores de edad intra o finioligocena. Encajada en la misma se encuentra una superficie poco extensa en las Sierras Exteriores y Marginales, sin relación con depósitos terciarios, a la que le asigna una posible edad mio-pliocena al situarse cronológicamente entre la superficie anterior y los aplanamientos pliocuaternarios situados a su pie. RonRícuez Vidal, J. (1982), en su estudio del karst de Guara considera que la doble superficie de Barrere es el mismo nivel deformado. 
Con posterioridad a la superficie finimiocena de BARrere o miopliocena de Peña se produjo el vaciado parcial de los materiales miocenos de la Depresión del Ebro entre el frente pirenaico y las plataformas calizas del Neógeno superior. Estas circunstancias vienen manifestadas por el hecho de que las plataformas de Almudévar y Fraga constituyeron barreras para la sedimentación pliocuaternaria. Además el extenso afloramiento de pliocuaternario del área de Candasnos, arranca de las plataformas calizas del Neógeno superior de la Sierra de Ontiñena. Todas estas observaciones ponen de manifiesto que en épocas pliocuaternarias existía una depresión de contacto entre las plataformas calizas y las Sierras Exteriores. Por otra parte la sedimentación pliocuaternaria es consecuencia de una etapa diastrófica que dio lugar a la elevación de las sierras pirenaicas y al hundimiento relativo de la Depresión del Ebro, instalándose un conjunto de abanicos aluviales (fig. 4), alimentados en nuestro caso a partir de sierras pirenaicas, de gran extensión pero de muy poca potencia, excepto en las áreas apicales donde la acumulación es mayor. Los ápices de estos abanicos se sitúan, por lo general, en la salida hacia la Depresión del Ebro de los ríos actuales pirenaicos, aunque en una posición más septentrional que la actual, ya que el propio pliocuaternario se apoya en su nivel superior sobre las sierras marginales.

Se reconocen tres niveles pliocuaternarios encajados unos en otros y correspondientes a diferentes etapas de instauración de abanicos aluviales. No tenemos ningún argumento que permita precisar si estos encajamientos se deben a causas climáticas o tectónicas.

El nivel inferior parece marcar, sobre todo en el interfluvio Cinca-Gállego, el comienzo del encajamiento y jerarquización de la red fluvial cuaternaria, dado que este últímo nivel (en este área señalada) adopta una morfo. logía de terrazas en las arterias principales, sobre todo en los ríos Alcanadre y Guatizalema. A partir de este momento comienza el desarrollo de los sistemas de terrazas cuaternarias. También entonces el río Noguera Ribagorzana dejó su disposición confluyente con el Cinca y fue capturado por un afluente del río Segre, antes ya de depositarse las terrazas cuaternarias más antiguas.

Dentro del Sector central de la Depresión del Ebro, en el piedemonte de la Cordillera Ibérica, el pliocuaternario presenta un menor desarrollo.

IBÁÑEz, M. J. (1976) en el piedemonte bajoaragonés diferencia un nivel elevado al que le asigna una edad Cuaternaria antigua. En el área de Ĺongares, Mensua, S. e Ibáñez, M. J. (1977) y Soriano, M. A. (1982) describen un nivel pliocuaternario con características de raña. Finalmente, Pellicer, F. (1980) en el piedemonte del Moncayo distingue dos niveles: el superior, finiterciario y retocado en el Villafranquiense y el inferior, del cuaternario antiguo, ambos modelados en glacis. En la actualidad no existen criterios para efectuar una correlación entre los niveles pliocuaternarios existentes en ambos piedemontes de la Depresión. 


\section{B I B L I O G R A F I A}

Barrere, P. (1951). - "La morphologie des Sierras Oscenses". Act. I. Congr. Int. Estud. Pirenaicos, t. 5, secc. 4, pp. 51-79, 9 figs., 20 pl., 2 mapas. San Sebastián. Publ. Inst. Estud. Pirenaicos. Zaragoza.

Barrere, P. (1962). - "Reliefs murs perches de la Navarre Orientale". Rev. Geogr. des Pyrenees et Sud-Ouest., t. XXXIII, pp. 36-40,

Bomer, B. (1957). - "Le Piemont de Huesca. Livret-guide de l'excursion Pyrenees". V. Congr. Int. "INQUA". Madrid.

Bомer, B. (1979). - “Les piedmonts du Bassin de l'Ebre (Espagne)". Mediterranée, núm. 3, pp. 19-25.

Crusafont, M., Villalta, J. F. y Riba, O. (1957). - "Villafranchien de Villarroya. Livret. guide de l'excursion núm. 3". V Congr. Int. "INQUA", pp. 11-15. Madrid- Barcelona.

IBÁÑEz, M. J. (1976). - "El p'edemonte ibérico bajoaragonés. Estudio Geomorfológico". Instituto de Geografia Aplicada C. S. I. C., 523 págs. Madrid.

Mensua, S. e Ibáñez, M. J. (1977). - "Terrazas y Glacis del Centro de la Depresión del Ebro". III Reunión Nac. Grupo Trabajo Cuaternario, pp. 1-18. Zaragoza.

Panzer, W. (1926).: - "Talentwicklung und Eiszeitklima in nordostlichen Spanien". $A b h$. der Sencken. Natur. Geseelschaft, t. 39, fasc. 3, Francfurt. (Trad. esp. de C. V.: EI desarrollo de los valles y el clima de la época Cuaternaria en el NE de España. - Est. Geográficos, núm. 30, pp. 79-130, Madrid, 1948.

Pellicer, F. (1980). - "El periglaciarismo del Moncayo". Geographicalia, 7-8, pp. 3-25. Zaragoza.

Peña Monne, J. L. (1978). - "Estudio geomorfológico de las sierras exteriores pirenaicas en la Zona de Oliana (prov. de Lérida)". Estudios Geológicos, vol. 34, pp. 99-106.

Peña, J. L. (1979). - "Consideraciones geomorfológicas sobre la terminación oriental del Montsec (Sierras Exteriores Pirenaicas, prov. de Lérida)",. Geographica, t. XXI-XXII, pp. 155-161.

Peña, J. L. (1980). - "Estudio geomorfológico de la Conca de Tremp y las Sierras Prepirenaicas leridanas entre los ríos Segre y Noguera Ribagorzana". Tesis Doctoral Fac. Filo.

sofia y Letras, 577 págs. Zaragoza.

Puignefábregas, C. (1975). - "La sedimentación molásica en la cuenca de Jaca". Monografías del Inst. Est. Pirenaicos, núm. 104, número extraordinario de Pirineos, 188 págs., 31 figs., 141 fot., 1 mapa.

Quirantes, J. (1978). - "Estudio sedimentológico y estratigráfico del Terciario continental de los Monegros". Institución Fernando el Católico, C. S. I. C. Zaragoza, 200 págs.

Rodriguez Vidal, J. (1981). - "Evolución geomorfológica de la Hoya de Huesca durante el Cuaternario". V Reunión Nac. Grupo Trabajo Cuaternario, pp. 258-266. Sevilla.

RonRícuez Vidal, J. (1982). - "El modelado kárstico de la Sierra de Guara: Dolinas de Cupierlo : Reunión Monográfica sobre el karst. Larra (en prensa).

Solé Sabarís, L. (1953). - "Terrazas cuaternarias deformadas de la cuenca del Ebro". Mem. R. Acad. Ciencias y Artes de Barcelona, 31 (7), pp. 239-259.

Solé Sabarís, L. y Font Tullot, J. M. (1929). - "Las terrazas del Segre en las inmediaciones de Lérida". Publ. Inst. Geol. Dip. prov. Barcelona, t. 2, 7 págs., 2 figs., 5 lám. Barcelona.

SorinNo, A. (1982). - "Investigaciones geomorfológicas en la región de Muel-Fuendetodos (prov. de Zaragoza)". Tesis de Licenciatura, Facultad de Ciencias, 123 p. Zaragoza. 


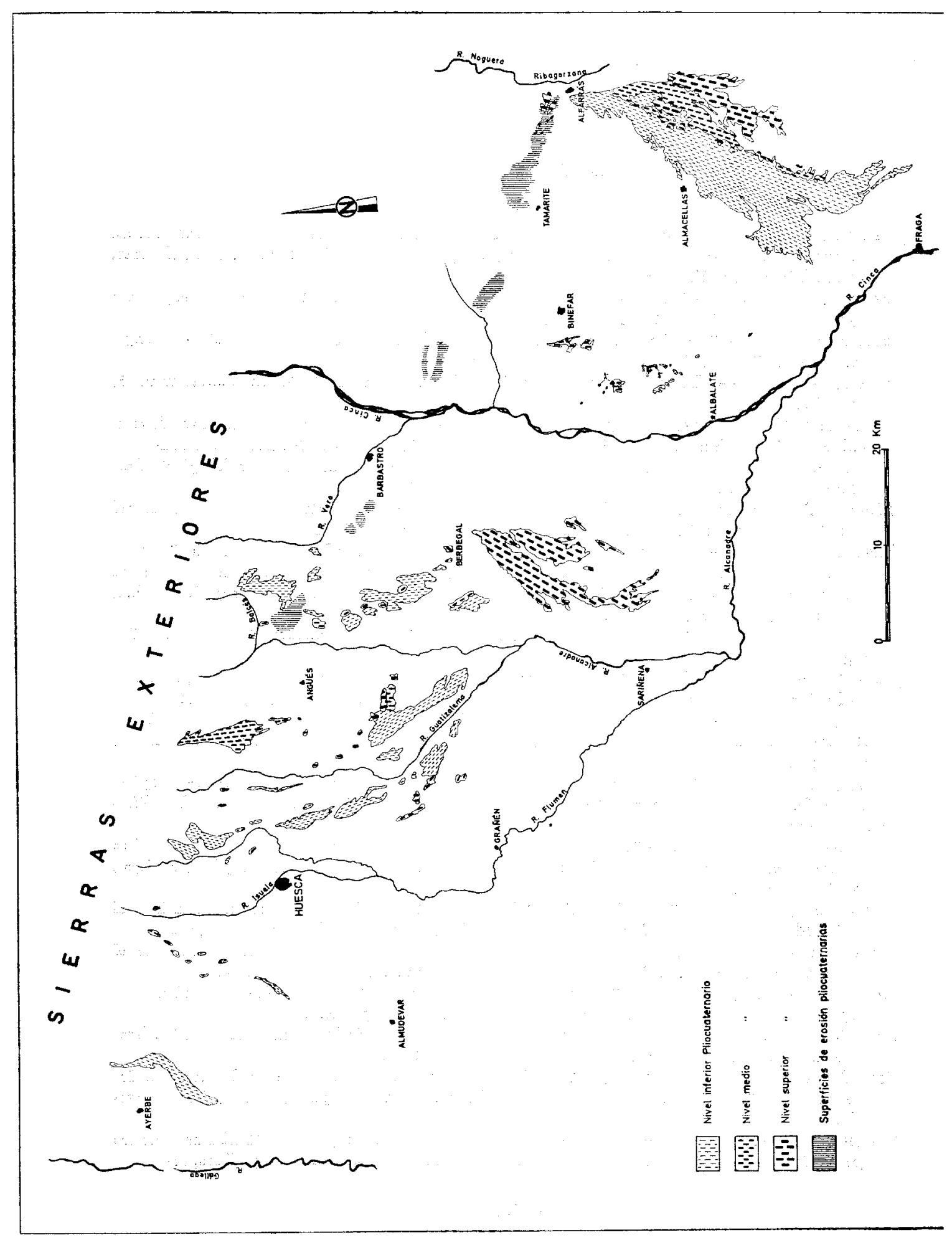

